\title{
Low parental care as a risk factor to lifetime depression in a community sample
}

\author{
Gordon Parker ${ }^{\mathrm{a}, *}$, Dusan Hadzi-Pavlovic ${ }^{\mathrm{a}}$, Steven Greenwald ${ }^{\mathrm{b}}$, Myrna Weissman ${ }^{\text {b,c }}$ \\ " Mood Disorders Unit, Prince Henry Hospital, and School of Psychiatry. University of New South Wales, Sydney. \\ New South Wales, Australia \\ ${ }^{\mathrm{b}}$ Division of Clinical and Genetic Epidemiology, New York State Psychiatric Institute, New York, NY, USA \\ 'Department of Psychiatry, College of Physicians and Surgeons of Columbia University, New York, NY, USA
}

Received 27 October 1993; revised 7 September 1994; accepted 7 September 1994

\begin{abstract}
A number of studies have reported links between experiencing low parental care and subsequent depressive experience. As the majority have involved patient samples, links may reflect anomalous parenting disposing to help-seeking behaviour (and patient status) rather than directly to depression. We, therefore, report a community study, so redressing any such artefact emerging from a patient sample and, additionally, quantify the relevance of low parental care to depression in comparison to several other risk factors (i.e., age, gender, educational level, socioeconomic status and marital status). Subjects were drawn from the ECA study and comprised those assessed at the 1-year follow-up interview undertaken at the New Haven site, with parental care being assessed by a key item from the Parental Bonding Instrument (PBI). Those reaching criteria for a lifetime episode of major depressive disorder were significantly more likely to report low care from both parents as well as to be female, divorced or separated, and younger. Low parental care (along with age and mental status but not female sex) appeared pathoplastic in being linked with an increased chance of psychopathology in general, rather than demonstrating specificity to major depressive disorder.
\end{abstract}

Keywords: Depression; Risk factor; Epidemiology; Parental characteristic

\section{Introduction}

It has long been proposed that aspects of the parent-child relationship dispose the child to depression in adulthood. While the roles of a parent can be variably defined, theoretical descriptions have emphasised the provision of a secure affec-

\footnotetext{
* Corresponding author. Address: Psychiatry Unit, The Prince of Wales Hospital, Randwick, New South Wales 2031, Australia. Fax: (61) (2) 398-7783.
}

tional base with the parent being both available and responsive as well as having the capacity to allow and encourage the child to move progressively away from that parental base to achieve social competence (Bowlby, 1977). Such dimensions of 'care' and 'encouragement of independence' are central to most measures of parental style (Gerlsma, 1990) and in their inverted form (i.e., parental indifference or rejection; and overprotection) have been held to dispose strongly to depression in adulthood. 
That central proposition has been investigated in some detail over the last decade, with two measures of parental care and overprotection (the Parental Bonding Instrument, PBI; and the Egna Minnen Betraffande Uppfostran, EMBU) being the most commonly used and with some consistency in findings (Parker, 1992). As items from the former measure are used in the current study, a brief overview of relevant research is provided.

The PBI (Parker et al., 1979) is a 25-item self-report measure, with subjects scoring parental attitudes and behaviours as remembered for their first 16 years, with scores generated for parental 'care' and parental 'protection'. A number of studies of nonclinical groups (Parker, 1983a) have demonstrated consistent associations (although not always significant) linking higher state and trait depression levels with low parental care and with parental overprotection. More importantly, a large number of case-control studies have been completed, indicating distinct specificity, with anomalous parenting (as defined and measured by the PBI) being irrelevant to both unipolar (Parker et al., 1987; Parker and Hadzi-Pavlovic, 1992) and bipolar (Parker, 1979; Joyce, 1984) expressions of melancholia but distinctly overrepresented in four case-control studies of nonmelancholic depressed patients (Parker, 1992), with the latter reporting significantly less parental care and somewhat more parental protection. Additive effects were examined and demonstrated in another case-control study of nonmelancholic depressives (Parker and HadziPavlovic, 1992), with the risk of depression being raised somewhat by reporting exposure to anomalous parenting from one parent and considerably by reporting such parenting from both parents.

Noncausal explanations for links between PBI scores and nonmelancholic depression have been considered to varying degrees. The possibility that depressed patients might merely score their parents negatively as a consequence of a depressed mood state or because of some general tendency to rate significant interpersonal relations in a negative way has been rejected in a series of studies (Parker, 1992). The possibility of a spurious association determined by a common genetic variable (e.g., a genetic influence, such as 'neuro- ticism', both increasing the chance of depression in a child and of a parent being uncaring and/or overprotective) was not supported in a study of adoptees (Parker, 1981), where links between their depression levels and PBI scores (for adopting parents) were as strong as demonstrated in nonclinical groups rating biological parents. More recently, this issue has been addressed more directly. Neale et al. (in press) required a sample of 1500 twins to complete both a significantly reduced (7-item) PBI questionnaire for each parent and a measure of state depression. While their modelling analyses found evidence for genetic determinants both of parenting style and of depression levels, they also established a significant link between parental style and depression.

While such findings are supportive of a causal process whereby low parental care disposes the child to later nonmelancholic depression, a caveat was raised by Mackinnon et al. (1989). They suggested that any association between nonmelancholic depression and earlier anomalous parenting might be restricted to those who seek help and who would, therefore, be overrepresented among psychiatric patients (i.e., the 'cases' in the reported case-control studies). Such a possibility has been examined indirectly in nonclinical groups where, as noted carlier, links have generally been established between PBI scores and state and trait depression levels.

A useful strategy for examining whether anomalous parenting leads more to help-seeking in depressed patients than to nonmelancholic depression per se is to study community samples, thereby overcoming any bias emerging from studying formal 'patients', whether inpatients or outpatients. Two such studies have produced contrasting findings. Mackinnon et al. (1989) assessed adults sampled from an electoral register in Canberra, Australia. Subjects completed the PBI at baseline and the depression section of the Delusions-Symptoms-States Inventory (Bedford and Foulds, 1978) on two separate occasions. The mean depression score (calculated from the two test occasions) was inter-correlated with $\mathrm{PBI}$ scores, with all coefficients being $<0.16$. Mean depression scores for those assigning parents to the varying PBI quadrants revealed only one sig- 
nificant result, with females being significantly more likely to assign their mothers to a low care quadrant.

The second study was undertaken as part of a general psychiatric community survey in Christchurch, New Zealand (Wells et al., 1989), and was a more direct test of the risk to onset hypothesis. A reinterview sample ( 90 chosen randomly and 74 who met DSM-III criteria for major depression in the previous year) completed the PBI. Those meeting criteria for depression scored both parents as significantly less caring and as more protective (Joyce, 1990), with the quantified case-control differences being in the same order as those reported in clinical groups. This study goes some way to rejecting the hypothesis that links between anomalous parenting and depression may be determined by a higher-order variable, help-secking behaviour.

We now report a second community study of depression 'caseness' (as against examining depression levels dimensionally), allowing an estimate of the risk of anomalous parenting to 'major depressive disorder' within a large general community setting independent of help-seeking. We focus on any risk effected by low parental care as the relevance of parental overprotection to nonmelancholic depression has been less clearly or inconsistently suggested - with Mackinnon et al. (1993) producing data from a large twin study to suggest that after 'low care' has been accounted for, 'overprotection' makes a minimal contribution to depression. In this study, the sample size and examination of other imputed or established risk factors to depression, such as age, scx, social class and marital status, allow the comparative importance of low parental care to be examined with some precision.

\section{Methods}

\subsection{Subjects}

The sample involved individuals surveyed in the NIMH Epidemiologic Catchment Area (ECA) program. The ECA study was a community survey of rates and risks for psychiatric disorders based on a probability sample of 18,571 adults aged $\geq 18$ years, living in five US communities (New Haven, CT; Baltimore, MD; St Louis, MO; Piedmont County, NC; and Los Angeles, CA). A full description of the purpose, methods and sampling frame can be found in Robins and Regier (1991). The data on parental style (see below) were obtained at the 1-year follow-up interview at the New Haven site - which included a multistage probability sample of community residents and was drawn from a 13-town area served by the Connecticut Mental Health Center, New Haven. The total number of subjects surveyed at that 1-year review was 3684 , representing $73 \%$ of the original sample interviewed and including an oversampling of persons aged $\geq 65$ years.

\subsection{Psychiatric assessment}

Each ECA site used the Diagnostic Interview Schedule (DIS; Robins et al., 1981), a highly structured interview designed for use by lay interviewers in epidemiologic field studies. Responses to the DIS questions are subjected to an algorithm generating DSM-III diagnoses (American Psychiatric Association, 1980) for the major psychiatric disorders. The disorder of focus in this report is the lifetime diagnosis of major depression, excluding a lifetime manic episode but may include grief for $\geq 1$ year. Other DIS/DSM-III lifetime diagnoses considered at the 1-year follow-up were dysthymia, bipolar disorder, panic disorder, schizophrenia or schizophreniform disorder, drug abuse, alcohol abuse, simple phobia, social phobia, agoraphobia, obsessive compulsive disorder, somatization disorder and antisocial personality disorder.

\subsection{Parental bonding measure}

Two central PBI items were selected for rating of each parent, with selection based on their strong factor loadings in the original PBI development studies (Fig. 1, Table 2, Parker et al., 1979) and because of their direct expression of the PBI scale constructs. One item assessed parental care ('As you remember your mother/ father in your first 16 years, how affectionate was she/he to you?') and the other assessed parental control ('How much did she/he try to control 
everything you did?'). Subjects taking part in the 1-year New Haven review were required to choose between 'very', 'moderately', 'slightly' or 'not at all' options in rating each parent. Checking either of the last two ratings assigned that parent to a 'low care' category and, if both parents were so rated, resulted in assignment to 'low parental care'. In these analyses, reported exposure to low parental care was examined in relation to (1) mothers alone, (2) fathers alonc and (3) mother and/or father.

Clearly, such an abbreviated measure would not be expected necessarily to mirror the full PBI measure, which has had extensive testing of its psychometric properties (e.g., Parker, 1983a), and so argued for some comparison of the brief measure against the full PBI measure in a sample allowing such comparative analyses. To that end, a subsample of 50 depressed subjects was sought from a larger sample of 123 depressed general practice and community depressives (Parker, 1993), with selection of the first fifty being consecutive apart from excluding subjects who were only able to rate one parent. As our present study considers only item 6 (the selected PBI 'care' item), we then intercorrelated (within our subsample of 50 depressives), item 6 care scores with the full PBI care scale scores. For mothers, the correlation coefficient was 0.83 ; for fathers, 0.86 , and for 'parental care' (summed parental item 6 scores vs. summed parental PBI care-scale scores) the coefficient was 0.83 . Further, subjects who checked the 'very' or 'moderately' choice in relation to item 6 (compared with those who instead checked 'slightly' or 'not at all'), returned significantly higher overall PBI total care-scale scores (mothers: 26.0 vs. $7.6, t=8.25$; fathers: 24.3 vs. $7.2, t=9.03$; both parents $=51.8$ vs. $27.5, t=7.02$; all $P<0.001$ ). These results indicate that item 6 responses in this study are likely to have provided an adequate representation of the complete PBI care scale.

\subsection{Statistical analyses}

To assess low parental care as a risk factor for presence or absence of a lifetime episode of major depression, three principal polytomous lo- gistic regression analyses were undertaken. The first assessed low parental care in those with major depression alone or comorbid with any other psychiatric disorder vs. those with no psychiatric disorder. The second compared those with major depression alone (i.e., without any comorbid disorder) vs. those with no psychiatric disorder. The third compared those with a diagnosis other than major depression vs. those with no psychiatric disorder.

In addition to quantifying the relevance of low parental care (compared with the other risk variables), we can examine their specificity to major depression. Thus, if any of the risk factors are not specific to major depression (and a risk to all disorders examined), we would expect to demonstrate links most strongly in the second analyses and less strongly for the first and third analyses, as the specificity effect would be attenuated to the extent of the risk factor also having links to the 'other diagnoses'. In such analyses, we examined the comparative influence of a number of demographic variables, each controlled for the other; female sex; younger age; educational level with last grade completed as a continuous variable, socioeconomic status ( 1 and 4 representing highest and lowest quartiles, respectively) and marital status (varying 'at risk' allocations being examined). Adjusted odds ratios (with 95\% confidence intervals) were derived, being adjusted for all other predictors in the equation since all predictors were entered simultaneously. The odds ratio indicates how much more likely (or less likely) a person in the comparison group (e.g., those with major depression) was to report the categorized risk variable (e.g., female sex and young age). A significantly increased chance of reporting the risk variable is indicated by an odds ratio $>1.0$ and by the lower end of the confidence interval being $>1.0$. A significantly decreased chance of reporting the risk variable is indicated by an odds ratio $<1.0$ and by the upper end of the confidence interval being $<1.0$.

\section{Results}

Of the 3684 subjects ( 2184 females and 1500 males), 176 (134 females and 42 males) or $4.8 \%$ 
met criteria for lifetime major depressive disorder (MDD) alone while 492 (358 females and 134 males) or $13.3 \%$ met criteria for MDD plus any other psychiatric diagnosis included in the ECA diagnostic list - rates representing wave three data from one site and containing both prevalence and incidence cases.

Mothers were rated as 'very' caring by $56 \%$, 'moderately' caring by $31 \%$, 'slightly' caring by $8 \%$ and 'not at all' caring by $4 \%$. For fathers, $41 \%$ were rated as 'very' caring, $35 \%$ 'moderately' caring, $16 \%$ 'slightly' caring and $8 \%$ 'not at all' caring. Overall then, mothers were rated as more caring than fathers $\left(\chi^{2}=211.4, \mathrm{df}=3, P<\right.$ $0.001)$.

Table 1 reports summary details for three separate analyses. In all analyses, several variables are consistently nonsignificant (i.e., education level, social class, middle age, being married, being a widow). In the first analysis, we seek to define the significant risk factors for those returning a diagnosis of MDD (alone or in conjunction with some other psychiatric diagnosis) compared with those without any psychiatric diagnosis. Being younger (i.e., both those aged 18-29 and those aged 30-44, compared with those $\geq 65$ years), being divorced or separated, being female and reporting low parental care (from mothers only, fathers only and from both parents) were the identified significant predictors. In the second analysis, we narrow the MDD subjects to those with MDD only (i.e., no other psychiatric diagnosis) and, when compared with those without any psychiatric diagnosis, we find four significant variables (female sex, being divorced or separated, being in the 18-29-year age group and reporting low paternal care). In the third analysis, contrasting those having MDD alone with those having any psychiatric diagnosis other than MDD, several variables (aged 18-44 years, male sex, being divorced or separated and both low paternal and low parental care) were significant.

We had, therefore, identified several variables (i.e., aged 18-29, sex, being divorced or separated and low paternal care) which were overrepresented in those who had either major depression only or any condition other than major depression compared with those without any diagnosis), suggesting that those risk factors may be pathoplastic - i.e., being a nonspecific risk factor to all

Table 1

Polytomous logistic regression analyses examining independent contribution of parental care and other risk factors to MDD

\begin{tabular}{|c|c|c|c|}
\hline $\begin{array}{l}\text { Risk factor } \\
\text { (odds calculated in reference to } \\
\text { first-mentioned category) }\end{array}$ & $\begin{array}{l}\text { Odds favouring MDD } \\
\text { alone or with another } \\
\text { diagnosis over no diagnosis }\end{array}$ & $\begin{array}{l}\text { Odds favouring } \\
\text { MDD alone } \\
\text { over no diagnosis }\end{array}$ & $\begin{array}{l}\text { Odds favouring } \\
\text { a non-MDD diagnosis } \\
\text { only over no diagnosis }\end{array}$ \\
\hline \multicolumn{4}{|l|}{ Age } \\
\hline $18-29$ vs. $65+$ & $2.0(1.2-3.5)$ & $2.7(1.4-5.3)$ & $3.5(2.4-5.0)$ \\
\hline $30-44$ vs. $65+$ & $2.1(1.4-3.3)$ & $1.6(0.9-2.8)$ & $1.5(1.1-2.1)$ \\
\hline $45-64$ vs. $65+$ & $0.9(0.6-1.5)$ & $1.4(0.8-2.5)$ & $0.7(0.5-1.1)$ \\
\hline Education & $0.9(0.8-1.0)$ & $1.1(0.9-1.3)$ & $0.9(0.9-1.0)$ \\
\hline \multicolumn{4}{|l|}{ Social class } \\
\hline SES 1 vs. 4 & $0.5(0.3-1.2)$ & $1.1(0.4-3.1)$ & $1.3(0.8-2.1)$ \\
\hline SES 2 vs. 4 & $1.3(0.9-1.9)$ & $0.9(0.5-1.5)$ & $1.1(0.8-1.4)$ \\
\hline SES 3 vs. 4 & $1.2(0.8-1.9)$ & $1.1(0.6-1.8)$ & $0.8(0.6-1.0)$ \\
\hline $\operatorname{Sex}(F)$ & $1.7(1.3-2.2)$ & $2.4(1.7-3.5)$ & $0.7(0.6-0.9)$ \\
\hline \multicolumn{4}{|l|}{ Marital status } \\
\hline Married/de facto vs. other & $0.8(0.6-1.2)$ & $1.2(0.7-1.9)$ & $1.1(0.8-1.4)$ \\
\hline Widowed vs. other & $1.1(0.7-1.8)$ & $1.2(0.6-2.3)$ & $1.5(1.1-2.1)$ \\
\hline Divorced/separated vs. other & $2.6(1.6-4.1)$ & $2.4(1.3-4.5)$ & $1.6(1.1-2.3)$ \\
\hline \multicolumn{4}{|l|}{ Parental care } \\
\hline PBI low care - mother & $1.9(1.2-2.9)$ & $1.3(0.7-2.5)$ & $1.3(0.9-1.9)$ \\
\hline PBI low care - father & $1.8(1.3-2.5)$ & $1.6(1.1-2.4)$ & $1.4(1.1-1.7)$ \\
\hline PBI low care - both parents & $2.0(1.3-3.2)$ & $1.6(0.9-2.9)$ & $1.5(1.1-2.1)$ \\
\hline
\end{tabular}

Significant odds ratios in bold. 
Table 2

Polytomous logistic regression analysis examining independent contribution of parental care and other risk factors to major depression alone vs. to a non-MDD diagnosis only

\begin{tabular}{ll}
\hline $\begin{array}{l}\text { Odds favouring first-mentioned } \\
\text { category }\end{array}$ & $\begin{array}{l}\text { Odds favouring } \\
\text { MDD alone over } \\
\text { a non-MDD } \\
\text { diagnosis only }\end{array}$ \\
\hline Age & $1.3(0.6-2.5)$ \\
$65+$ vs. 18-29 & $0.9(0.5-1.7)$ \\
$65+$ vs. 30-44 & $\mathbf{0 . 5}(\mathbf{0 . 3 - 1 . 0 )}$ \\
65 + vs. 45-64 & $\mathbf{0 . 8}(\mathbf{0 . 7}-1.0)$ \\
Education & $1.2(0.4-3.4)$ \\
Social class & $1.3(0.7-2.3)$ \\
SES 4 vs. 1 & $0.7(0.4-1.3)$ \\
SES 4 vs. 2 & $\mathbf{3 . 4}(\mathbf{2 . 3 - 5 . 0 )}$ \\
SES 4 vs. 3 & $1.1(0.6-1.8)$ \\
Sex (F) & $0.8(0.4-1.6)$ \\
Marital status & $1.5(0.8-2.9)$ \\
Married/de factor vs. other & \\
Widowed vs. other & $1.0(0.5-2.0)$ \\
Divorced /separated vs. other & $1.2(0.8-1.8)$ \\
Parental care & $1.1(0.6-2.0)$ \\
PBI low care - mother &
\end{tabular}

Significant odds ratios in bold.

disorders. We, therefore, undertook a subsidiary analysis contrasting those positive for major depression only with those positive only for a diagnosis other than major depression. Table 2 shows that those with major depression were far more likely to be female and somewhat less likely to bc in the 45-64-year age band and to have less education. None of the PBI measures differentiated the two groups, indicating that PBI care was not a specific risk variable to major depression alone.

\section{Discussion}

We tested the hypothesis that low parental care is a significant risk factor to lifetime depression per se and not merely to help-seeking (i.e., patient/treatment status) in those who develop depression, a hypothesis requiring a community or nonclinical sample. While demands on respondents' time disallowed use of the complete PBI measure, our separate analysis of an independent sample indicated that the PBI care scale item used in this study captured the PBI care domain extremely well, with coefficients of $>0.83$ for the three 'parental' measures and with tests of our dichotomized 'high-care' and 'low-care' allocations well supported. Greater concern should perhaps be expressed about implications of the sample size, in that the large numbers of subjects present a risk of a type 2 error. Thus, statistically significant variables may or may not be clinically significant, a caveat that should be considered in reviewing our summarised positive findings. Study findings confirm the link suggested in many clinical groups, with those reaching criteria for a lifetime episode of MDD (ignoring comorbidity for the moment) being significantly more likely to report low care from both parents.

Additionally, the ECA study design allowed us to quantify the comparative importance of low parental care as a risk factor to major depression (i.e., its strength in comparison to recognized risk factors) and to examine for any specificity of low parental care to MDD. One of the key strengths of the ECA study design emerges from its capacity to quantify macroscopic risk factors with some confidence (as a consequence of the large sample size), so encouraging a more efficient approach to deciding the risk variables that should be pursued in both more refined studies and in subsequently providing theoretical explanations.

While results were influenced by comorbidity issues and choice of comparison groups, the study confirms that low parental care is worthy of inclusion in any list of established risk factors to depression, being relevant after adjusting for a number of recognized major risk factors, such as female sex, younger age and divorce/separation. Our analyses indicated, however, that low parental care is unlikely to be a risk factor with specificity to major depression alone. Thus, in our Table 2 analyses, where the comparison group was those positive for a disorder other than major depression, low parental care was no longer significant. The same phenomenon held for several other risk factors (i.e., younger age and divorced or separated), indicating that they are better viewed as nonspecific or pathoplastic risk factors disposing to a variety of psychiatric disorders and not just to major depression alone. 
The sex of the subject showed a different effect. Females were significantly overpresented in the major depression group (alone or when those with comorbid conditions were included) and underrepresented in the residual heterogeneous diagnostic group. Thus, when those two groups were compared (Table 2), female sex not only retained its specificity to major depression but its overrepresentation was even more distinct.

The validity of the PBI (both as a measure of perceived and of actual parenting) has been examined in a number of studies (e.g., Parker, 1983a, 1992) with generally supportive findings offering some indirect support for a causal process linking an increased chance of adult depression in those exposed to uncaring parenting. More importantly, the twin study noted earlier (Neale et al., in press) did confirm, in addition to identifying genetic influences on parenting and on depression, a link between parental style and depression. Other studies have demonstrated that PBI scores predict risk both to onset of depression (e.g., Boyce et al., 1991) and to course of depression (Gotlib et al., 1988). The present study not only confirms the comparative importance of low parental care as a risk factor to adult depression and its salience within the general community but suggests both its comparative and independent importance when considered in conjunction with other recognized risk factors. Equally importantly, we established that low parental care (along with several other risk factors) is not specific to major depression, an issue not generally appreciated in studies which do not examine for cffects emerging from comorbidity.

\section{Acknowledgments}

In the ECA program, the NIMH principal collaborators were D.A. Regier, B.Z. Locke and J.D. Burke, Jr. The project officers were C.A. Traub and W. Huber. The principal and co-investigators at the five sites were: Yale University J.K. Myers, M.M. Weissman and G.L. Tischler; Johns Hopkins University - M. Kramer, S. Shapiro and E. Gruenberg; Washington University - L.N. Robins and J. Helzer; Duke Univer- sity - L. George and D. Blazer; and University of California at Los Angeles - M. Karno, R. Hough, J. Escobar, A. Burnam and D. Timbers. This study was supported in part by NIMH Grant 5RO1 MH 43525-02 and by a grant from Upjohn Pharmaceutical Company. We also thank A. Sengoz for additional data analysis assistance.

\section{References}

Amcrican Psychiatric Association (1980) Diagnostic and Statistical Manual of Mental Disorders. 3rd Ed. American Psychiatric Association, Washington, DC.

Bedford, A. and Foulds, G.A. (1978) Manual of the Delusions-Symptoms State Inventory (State of Anx iety-Depression). NFER, Windsor.

Bowlby, J. (1977) The making and breaking of affectional bonds. Br. J. Psychiatry 130, 201-210.

Boyce, P., Hickie, I. and Parker, G. (1991) Parents, partners or personality? Risk factors for post-natal depression. J. Affect. Disord. 21, 245-255.

Gerlsma, C., Emmelkamp, P.M. and Arrindell, W.A. (1990) Anxiety, depression, and perception of early parenting: a meta-analysis. Clin. Psychol. Rev. 10, 251-277.

Gotlib, 1.H., Mount, J.H., Cordy, N.I. and Whiffen, V.E. (1988) Depression and perceptions of early parenting: a longitudinal investigation. Br. J. Psychiatry 152, 24-27.

Joyce, P.R. (1984) Parental bonding in bipolar affective disorder. J. Affect. Disord. 7, 319-324.

Joyce, P.R. (1990) A community study of atypical depression. Paper presented at the Section of Epidemiology and Community Psychiatry, Toronto, June 1990.

Mackinnon, A.J., Ilenderson, A.S., Scott, R. and DuncanJones, P. (1989) The parental bonding instrument (PBI): an epidemiological study in a general population sample. Psychol. Med. 19, 1023-1034.

Mackinnon, A., Henderson, A.S. and Andrews, G. (1993) Parental 'affectionless control' as an antecedent to adult depression: a risk factor refined. Psychol. Med. 23. 135141.

Neale, M.C., Walters, F., Heath, A.C., Kessler, R.C., Perusse, D., Eaves, L.J. and Kendler, K.S. (in press) Depression and parental bonding: cause, consequence, or genetic covariance? Gen. Epidem.

Parker, G. (1979) Parental characteristics in relation to depressive disorders. Br. J. Psychiatry 134, 138-147.

Parker, G. (1981) Parental reports of depressives: an investigation of several explanations. J. Affect. Disord. 3, 131140.

Parker, G. (1983a) Parental Overprotection: A Risk Factor in Psychosocial Development. Grune and Stratton, New York, NY.

Parker, G. (1983b) Parental 'affectionless control': a risk factor defined. Arch. Gen. Psychiatry 40, 956-960. 
Parker, G. (1992) Early environment. In E.S. Paykel (Ed.), Handbook of Affective Disorders. 2nd Ed. Guilford Press, New York, NY.

Parker, G. (1993) Parental rearing style: examining for links with personality vulnerability factors for depression. Soc. Psych. Psych. Epidemiol. 28, 97-100.

Parker, G. and Hadzi-Pavlovic, D. (1992) Parental representations of melancholic and non-melancholic depressives: examining for specificity to depressive type and for evidence of additive effects. Psychol. Med. 22, 657-665.

Parker, G., Kiloh, L. and Hayward, L. (1987) Parental representations of neurotic and endogenous depressives. J. Affect. Disord. 13, 75-82.
Parker, G., Tupling, H. and Brown, L.B. (1979) A parental bonding instrument. Br. J. Psychol. 52, 1-10.

Robins, L.N., Helzer, J.B. and Croughan, J.L. (1981) The NIMH diagnostic interview schedule: its history, characteristics, and validity. Arch. Gen. Psychiatry 39, 381-389.

Robins, L.N. and Regier, D.A. (1991) Psychiatric Disorders in America: The Epidemiologic Catchment Area Study. The Free Press, New York, NY.

Wells, J.E., Bushnell, J.A., Hornblow, A.R., Joyce, P.R. and Oakley-Browne, M.A. (1989) Christchurch psychiatric epidemiology study, part I: methodology and lifetime prevalence for specific psychiatric disorders. Aust. N.Z. J. Psychiatry $23,315-326$. 